\title{
INFLUENCE OF SINGLE WHOLE BODY VIBRATION TRAINING UNIT ON KINEMATICS OF HUMAN GAIT IN CHILDREN WITH NEUROLOGICAL DISORDERS
}

Utilisation of Whole body vibration (WBV) has been considered as a beneficial and powerful method to improve mobility and physical status of the patients during past decade. Benefits that were already published include enhanced muscular strength, power or bone density. However, there were also no significant effects reported in several clinical studies. The main aim of this pilot study was to investigate immediate effects of single WBV training unit on quality of gait kinematics in children patients suffering from neurological disorders. Five children ( $4.10 \pm 1.75$ years, 1 male and 4 females) were assessed before WBV exposure (10 minutes, $2 \mathrm{~mm}$ vertical vibrations, $30 \mathrm{~Hz}$ ) and then 1 minute afterwards. Individual changes in gait parameters were analysed and the results indicated significant positive effects of WBV in two patients. Other two patients registered positive changes, but these changes were not statistically significant. One of the patients included in this pilot study registered worsening of gait symmetry.

Keywords: Gait Analysis, whole-body vibration, rehabilitation.

\section{Introduction}

Whole body vibration (WBV) represents a concept that was applied in several studies to confirm benefits for astronauts, athletes, and wellness of healthy population [1 - 3]. Positive results were obtained also in clinical studies in patients with various diseases [4 - 7]. WBV has been also studied for its dangerous effects on humans, especially when exposed as occupational vibration at high amplitudes and specific frequencies [8 - 10].

Recent clinical works suggest that low amplitude and low frequency of mechanical stimulation of the human body is a safe and effective way to exercise musculoskeletal structures [11] The studies realized during past decade indicate that WBV may increase muscle strength, neuromuscular function, bone mass and mineral density [12], can be useful in improving physical capacity, cardiorespiratory functions, hormonal production, proprioception, and balance [13 - 14]. Despite of WBV positive effects presented in almost all related research studies, the authors interpret their results with caution. Also, the underlying mechanisms by which WBV enhance neuromuscular performance vary between studies and are still unclear. Inconsistency in presented results is caused by various training protocols and heterogeneity in study designs.

Because of both the positive and the negative effects of WBV on human body and its systems, it is important to consider all loading parameters that may affect WBV benefits. These parameters include type of vibration, frequency, amplitude, direction and exposure time, but also the position and activity of the subject on the WBV platform. The effect of vibration may be also tested using various modelling techniques [15 - 19].

The aim of this study was to investigate whether the single WBV session has any positive effects on gait kinematics in children patients. This was based on assumption that the WBV may stimulate muscle activation and that application of WBV will result in improvement of human gait quality.

\section{Material and methods}

A group of five children patients (age $4.10 \pm 1.75$ years, 1 male, 4 females) with hemiparesis or paraparesis of lower extremities were included in this pilot study. No of the patients had prior experience in WBV training. All patients and their parents or legal representatives were informed about the WBV training, tests to be realized and possible risks and benefits of the research. Prior to participation they gave written informed consent approved together with the study design by the local Ethics Committee.

All participants attended a familiarization session before the study was realized. No other physical treatment or intervention was realized at least 24 hour before WBV session. The anthropometric measures were also taken and registered in patients' experimental

\footnotetext{
* ' Jaroslav Majernik, ${ }^{2}$ Jozef Zivcak

'Department of Medical Informatics, Faculty of Medicine, Pavol Jozef Safarik University in Kosice, Slovakia, E-mail: jaroslav.majernik@upjs.sk

${ }^{2}$ Department of Biomedical Engineering and Measurement, Faculty of Mechanical Engineering, Technical University in Kosice, Slovakia
} 
protocols. As for the aim of the study, the anthropometric characteristics of lower extremities were preferred, including thigh length (right: $21.80 \pm 5.02 \mathrm{~cm}$, left: $21.60 \pm 4.88 \mathrm{~cm}$ ), calf length (right: $23.80 \pm 3.83 \mathrm{~cm}$, left: $23.40 \pm 3.63 \mathrm{~cm}$ ) and foot length (right: $15.40 \pm 0.89 \mathrm{~cm}$, left: $15.20 \pm 0.84 \mathrm{~cm}$ ).

The experimental protocol was designed to discover potential immediate response of a single WBV training unit to the quality of gait kinematics in children patients. Training sessions were supervised by rehabilitation specialist and measurements as well as WBV sessions were conducted in the same thermally neutral room intended for physical training. All subjects did not engage in any therapeutic or rehabilitation procedures before testing.

The training session started with physical examination and short warming-up walk. Then, the patient's gait was captured and analysed before WBV exposure. Participants were asked to walk at their natural waking speed along the $6 \mathrm{~m}$ long path. After reaching the end point of the path, they were asked to turn back $\left(180^{\circ}\right)$, i.e. change the direction of gait, and to walk back to the starting point. Then, they turned back again and walked to the end point of the path, where the last turn back was realized and the patients finished walking in starting point of the path. In that sense, the subjects passed the length of walking path four times. WBV session followed one minute after this control gait was realized and captured. Here, each participant stood in static position on the vibration platform (VibroGym inSPORTline) with no shoes and socks and holding on the device handle. Erected posture with slightly bended knees was required during vibration test. The patients were asked to stop the training in the case of any pain responses to vibration. Duration of one WBV training unit was set to 10 minutes. Synchronous vertical stimulus with frequencies from $25-45 \mathrm{~Hz}$ and amplitude of $1-2 \mathrm{~mm}$ results in a significant increase in leg muscle activity as measured via electromyography. Therefore, the sinusoidal vertical vibration frequency was set to $30 \mathrm{~Hz}$ with amplitude of $2 \mathrm{~mm} .1$ minute rest interval followed after this WBV exposure. Then, the patient's gait was captured and analysed again.

Gait assessment was performed using our marker-free motion analysis system MAFRAN. Here, the patient's gait in sagittal plane is captured using any commercial video camera. Then, the raw record is used in the system to reconstruct motion trajectories of human body anatomical landmarks, i.e. the trajectories of all lower extremity joints and adjacent segments. These trajectories are consequently used to calculate other kinematical parameters for detailed description of patient's gait. Here analysed parameters include positions, velocities and accelerations of individual joints, hip flexion/extension, knee flexion/extension, and ankle plantar/ dorsal flexion angles, gait cycle length, gait cycle time, gait cycle velocity, cadence (cycles per minute), stance phase and swing phase of the gait cycle.

All the parameters were analysed individually within the subject and then within the group of here included patients. The kinematical characteristics of the patients were evaluated as differences between right and left side. The hypothesis was based on assumption that these differences should be smaller after WBV training comparing values obtained before WBV training. Otherwise, the WBV will probably have no immediate benefits for gait kinematics. Statistical methods included descriptive statistics and Student's paired t-test and were used to ascertain specific and significant differences. The significance level was set to $\mathrm{P}>0.05$.

\section{Results}

All children patients accepted here realized WBV sessions very well. No one reported any pain or expressed any problems during WBV exposure. A first analysis was performed with anatomical joint angles of lower extremities in sagittal plane. Individually, no of the patients had the same curves of all tree
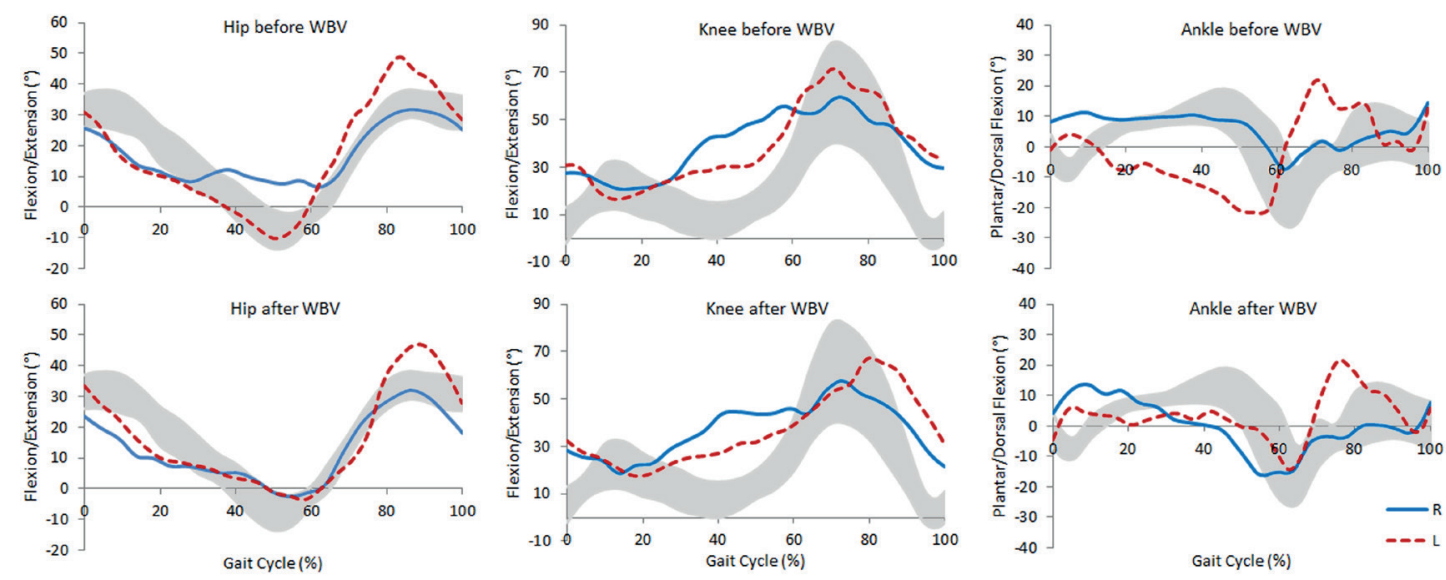

Fig. 1 Anatomical joint angles in 6 years old female patient with left paraparesis before and after WBV exposure (solid line - right leg, dashed line - left leg, greyed area - physiological gait values) 
joints comparing before and after WBV values. At least one of the anatomical joint angles was changed either in positive or negative direction. An example of anatomical joint angles changes in 6 years old female patient with left paraparesis is shown in Fig. 1.

A paired t-test determined that the mean decrease of differences $(\mathrm{M}=-1.88, \mathrm{SD}=5.099, \mathrm{~N}=51)$ was significantly greater than zero, $\mathrm{t}(50)=-2.63$, two-tail $\mathrm{p}=0.000(95 \% \mathrm{CL}$ $=1.434)$, providing evidence that the WBV was effective in reduction of differences between right and left hip flexion/ extension angles. Knee flexion/extension angles of the same patient showed that the mean differences between right and left side before and after WBV were changed, but the mean decrease of differences $(\mathrm{M}=2.17, \mathrm{SD}=8.213, \mathrm{~N}=51$ ) was not significantly greater than zero, $\mathrm{t}(50)=1.89$, two-tail $\mathrm{p}=0.065$ $(95 \% \mathrm{CL}=2.310)$, providing evidence that the WBV was not effective in reduction of differences between right and left knee flexion/extension angle in this patient. The mean decrease of the right and the left side differences in ankle plantar/dorsal flexion angles of the same 6 years old female patient $(\mathrm{M}=3.47, \mathrm{SD}=$ $4.858, \mathrm{~N}=51$ ) was significantly greater than zero, $\mathrm{t}(50)=5.10$, two-tail $\mathrm{p}=0.000(95 \% \mathrm{CL}=1.366)$ provided evidence that the WBV was effective in reduction of differences between right and left ankle plantar/dorsal flexion angle.

Anatomical joint angles were analysed in all participants of this study in the same way. The summary of right and left side differences in anatomical joint angles in children patients showed that the most significant changes were registered in the ankle plantar/dorsal flexion angle $(100.00 \%)$, followed by hip flexion/extension angle (80.00\%) and knee flexion/extension angle $(60.00 \%)$. However, these significant changes include both the positive and the negative changes. The only significant positive changes were recognized in ankle plantar/dorsal flexion angle $(80.00 \%)$, followed by the knee flexion/extension angle $(60.00 \%)$ and hip flexion/extension angle $(40.00 \%)$. Individually, there were two patients $(40.00 \%)$ who had at least two significant positive changes of these kinematical parameters or they had no significant negative changes (subjects 1 and 4). Two patients $(40.00 \%)$ had no beneficial improvements resulting from applied WBV exposure (subjects 2 and 3 ) and one $(20.00 \%)$ of the children patients registered worsening because of no significantly positive or only significantly negative changes (subject 5 ).
The second analysis was performed in spatio-temporal parameters. Here, the symmetry of all characteristics was examined and summarized. The mean differences between right and left side and standard deviations of these parameters are listed in Table 1.

None of here analysed parameters had significantly either positive or negative changes. Nevertheless, some of the parameters had positive and another negative tendency. The positive trends were shown in decreasing differences between right and left side in gait cycle length $(2.276 \pm 9,837)$, velocity $(0.008 \pm 0.121)$ and cadence $(0.416 \pm 3.565)$. Gait cycle time remained almost unchanged $(0.000 \pm 0.057)$. Negative trends were registered in gait cycle phases (stance: $-1.200 \pm 3.626$, swing $-1.188 \pm 3.630$ ).

\section{Conclusions}

The effect of single WBV training unit was tested in the group of children patients with hemiparesis or paraparesis in lower extremities. The results of this pilot study proved that WBV influences kinematics of human gait. Significant improvements were also confirmed in some of the here analysed parameters. On the other hand, changes in anatomical joint angles and in spatiotemporal characteristics, even if not significant had both the positive and the negative trends. To confirm the long-term effect the individually planned training should be designed. First of all, this training should respect limitations given by patient's disease. Then, the amplitude, frequency, duration and repetition of WBV during training unit will be adopted to the particular patient. The usage of WBV in patients' therapy, especially in patients with neurological disorders, has to be managed by physicians to avoid serious injuries, physical harms and/or other health related damages.

\section{Acknowledgements}

Results presented in this work were obtained with the support of the national grant KEGA 005UPJS-4/2012.

\begin{tabular}{|c|c|c|c|}
\hline & Pre & Post & Delta pre/post \\
\hline GC length $(\mathrm{cm})$ & $8.560 \pm 8.362$ & $6.284 \pm 4.595$ & $2.276 \pm 9,837$ \\
\hline GC time (s) & $0.088 \pm 0.072$ & $0.088 \pm 0.018$ & $0.000 \pm 0.057$ \\
\hline GC velocity (m/s) & $0.112 \pm 0.084$ & $0.104 \pm 0.043$ & $0.008 \pm 0.121$ \\
\hline Cadence (GC/min) & $7.258 \pm 5.147$ & $6.842 \pm 2.945$ & $0.416 \pm 3.565$ \\
\hline Stance phase (\%) & $2.784 \pm 0.756$ & $3.984 \pm 2.983$ & $-1.200 \pm 3.626$ \\
\hline Swing phase (\%) & $2.784 \pm 0.756$ & $3.972 \pm 2.990$ & $-1.188 \pm 3.630$ \\
\hline
\end{tabular}




\section{References}

[1] CARDinAlE, M., WAKELING, J.: Whole Body Vibration Exercise: Are Vibrations Good for You?”, Br J Sports Med, 2005, vol. 39, pp. 585-589.

[2] PRISBY, R., D., LAFAGE-PROUST, M., MALAVAL, L., BELLI, A., VICO, L.: Effects of Whole Body Vibration on the Skeleton and other Organ Systems in Man and Animal Models: What we know and what we need to know, Ageing Research Reviews, vol. 7, 2008, pp. 319-329.

[3] MADOU, K. H., CRONIN, J. B.: The Effects of Whole Body Vibration on Physical and Physiological Capability in Special Populations, Hong Kong Physiotherapy J., vol. 26, 2008, pp. 24-38.

[4] MilaneSE, C., PISCiTElli, F., SiMONI, C., PUGliarellO, R., ZANCANARO, C.: Effects of Whole-body Vibration with or without Localized Radiofrequency on Anthropometry, Body Composition, and Motor Performance in Young Nonobese Women, J. of Alternative and Complementary Medicine, 2012, 18 (1), pp. 69-75.

[5] GloecKl, R., HeinzelmanN, I., BAEUERle, S., DAMM, E., SCHWEDHELM, A.-L., DiRIL, M., BUHROW, D., JERRENTRUP, A., KENN, K.: Effects of Whole Body Vibration in Patients with Chronic Obstructive Pulmonary Sisease A Randomized Controlled Trial, Resp. Medicine, 2012, Vol. 106, pp. 75-83.

[6] Totosy de Zepetnek, J. O., Giangregorio, L. M., Craven, B. C., "Whole-body vibration as potential intervention for people with low bone mineral density and osteoporosis: A Review, J. of Rehabilitation Research and Development, vol. 46, No. 4, 2009, pp. 529-542.

[7] TORVINEN, S., KANNUS, P., SIEVANEN, H., JARVINEN, T. A. H., PASANEN, M., KONTULAINEN, S., JARVINEN, T. L. N., JARVINEN, M., OJA1, P., VUORI1, I.: Effect of Four-month Vertical Whole Body Vibration on Performance and Balance, Medicine \& Science in Sports \& Exercise, vol. 34, No. 9, 2002, pp. 1523-1528.

[8] SANTOS, B. R., LARIVIERE, CH., DELISLE, A., PLAMONDON, A., BOILEAU, P.-E., IMBEAU, D.: A Laboratory Study to Quantify the Biomechanical Responses to Whole-body Vibration: The Influence on Balance, Reflex response, Muscular activity and Fatigue, Intern. J. of Industrial Ergonomics, 38, 2008, pp. 626-639.

[9] MANI, R., MILOSAVLJEVIC, S., SUlLIVAN, S. J.: The Effect of Occupational Whole-body Vibration on Standing Balance: A Systematic Review, Int. J. of Industrial Ergonomics, vol. 40, 2010, pp. 698-709.

[10] MAEDA, S., MANSFIELD, N. J, SHIBATA, N.: Evaluation of Subjective Responses to Whole-body Vibration Exposure: Effect of Frequency Content, Intern. J. of Industrial Ergonomics, vol. 38, 2008, pp. 509-515.

[11] ARTERO, E. G., ESPADA-FUENTES, J. C., ARGUElles-CiEnfuegos, J., ROMAN, A., GOMEZ-LOPEZ, P. J., GUTIERREZ, A.: Effect of Whole-body Vibration and Resistance Training on Knee Extensors Muscular Performance, European J. of Applied Physiology, 2012, 112 (4), pp. 1371-1378.

[12] SLATKOVSKA, L., ALIBHAI, S. M. H., BEYENE, J., HU, H., DEMARAS, A., CHEUNG, A. M.: Effect of 12 Months of Wholebody Vibration Therapy on Bone Density and Structure in Postmenopausal Women: A Randomized Trial, Annals of Internal Medicine, 2011, 155 (10), pp. 668-679.

[13] ClAerbout, M., GEBARA, B., ILSBROUKX, S., VERSCHUEREN, S., PEERS, K., VAN ASCH, P., FEYS, P.: Effects of 3 Weeks' Whole Body Vibration Training on Muscle Strength and Functional Mobility in Hospitalized Persons with Multiple Sclerosis, Multiple Sclerosis, 2012, 18 (4), pp. 498-505.

[14] PENHAKER, M., KASIK, V., HRVOLOVA, B.: Advanced Bilirubin Measurement by a Photometric Method, Elektronika ir Elektrotechnika, 2013, 19 (3), pp. 47-50.

[15] RAKHEJA, S., DONG, R. G., PATRA, S., BOILEAU, P.-E., MARCOTTE, P., WARREN, C.: Biodynamics of the Human Body under Whole-body Vibration: Synthesis of the Reported Data, Intern. J. of Industrial Ergonomics, 2010, vol. 40, pp. 710-732.

[16] MACUROVA, A., MACURA, D.: Jacobian of the Generalized C-hyperbolic Coordinates, Intern. J. of Pure and Applied Mathematics, 2009, 53 (4), pp. 563-569.

[17] PUSTKOVA, R., KUTALEK, F., PENHAKER, M., NOVAK, V.: Measurement and Calculation of Cerebrospinal Fluid in Proportion to the Skull, Proc. of $9^{\text {th }}$ RoEduNet IEEE Intern. Conference, RoeduNet 2010, 2010, Article number 5541601, pp. 95-99.

[18] SAYENKO, D. G., MASANI, K., ALIZADEH-MEGHRAZI, M., POPOVIC, M. R., CRAVEN, B. C.: Acute Effects of Whole Body Vibration During Passive Standing on Soleus H-reflex in Subjects with and without Spinal Cord Injury, Neuroscience Letters, 482, 2010, pp. 66-70.

[19] ANDREJKOVA, J., SIMSIK, D., DOLNA, Z.: An Experience from Testing an Ambient Intelligence, Devices for Household - Case Study, SAMI $2010-8^{\text {th }}$ Intern. Symposium on Applied Machine Intelligence and Informatics, Proc. 2010, Article number 5423717, pp. 283-286. 